\title{
Ka-Band TDD Front-End with Gate Shunt Switched Cascode LNA and Three-Stack PA on 22nm FDSOI CMOS Technology
}

\author{
Mikko Hietanen, Jere Rusanen, Janne P. Aikio, Nuutti Tervo, Timo Rahkonen, Aarno Pärssinen \\ University of Oulu, Finland \\ $<$ firstname>.<lastname>@oulu.fi
}

\begin{abstract}
TDD Ka-band front-end with integrated switch on 22nm FDSOI CMOS technology is implemented for 5G NR at $24-28 \mathrm{GHz}$ bands. Shunt switch technique reduces front-end performance only by $2 \mathrm{~dB}$ at $24 \mathrm{GHz}$ compared to stand-alone reference amplifiers. Output power of TX with stacked PA is 13.6 $\mathrm{dBm}$ with $15 \mathrm{~dB}$ of peak gain, and $\mathrm{RX}$ front-end has $5 \mathrm{~dB}$ noise figure at $24 \mathrm{GHz}$, both measured at the antenna port. Maximum average channel power at $28 \mathrm{GHz}$ was $4.8 \mathrm{dBm}$ with $100 \mathrm{MHz}$ 64-QAM OFDM signal within 5G ACPR and EVM specifications. The PA and LNA amplifiers dissipate $183 \mathrm{~mW}$ and $4.6 \mathrm{~mW}$ from $2.8 \mathrm{~V}$ and $0.8 \mathrm{~V}$ supplies, respectively, occupying only $0.19 \mathrm{~mm}^{2}$.

Keywords - CMOS, LNA, mmWave, PA, SOI, TDD.
\end{abstract}

\section{INTRODUCTION}

Commercialization of 5 th generation communications (3GPP 5G NR) at low end of millimeter wave spectrum $(\mathrm{mmW})$ requires highly integrated, area efficient broadband phased arrays. Time division duplex (TDD) antenna front-ends (FE) using modern CMOS processes are susceptible to low breakdown voltages. Specific attention should be paid to low loss TX-RX switch that is a key element in the front-end. 22nm Fully Depleted Silicon-on-Insulator (FDSOI) technology is suitable solution for $\mathrm{mmW}$ integration with possibility to combine digital processing with high performance active and passive RF circuitry [1]. In addition, stacked PA structures favored in SOI based technologies allow higher power via higher supply voltages, but on the other hand can easily stress front-end switch beyond acceptable limits [2],[3].

High array gain to achieve decent link budget requires a large number of parallel antenna front-ends both in base station and in mobile devices. Therefore, a major portion of power of a wideband transceiver system is dissipated in radio front-end. In TDD systems, low power consumption and noise figure in the receiver are as important as decent efficiency and output power in the transmitter, especially in mobiles when there is more traffic in downlink direction. Therefore, TX-RX switching mechanism should have the lowest possible loss in both modes minimally loading the other operation mode. At $\mathrm{mmW}$ frequencies, such switch is not feasible to implement with a single switching transistor due to excessive parasitics and limited voltage handling capabilities of modern small gate length devices. In previous work, capacitive parasitics have been resonated out with inductors that add area penalty [4]. Alternative way is to use quarter-wave transformers to isolate
PA and LNA. However, those are even larger structures and are difficult to tune [5]. Common matching techniques have also been reported, however these tend to have complex and time consuming iterative process from closed form design equations to practical implementation [6],[7]. Nevertheless, a straight forward solution to implement a front-end without bulky and lossy switch structures would improve efficiency and reduce cost and design time significantly.

This work demonstrates a simple yet effective technique to combine LNA and stacked PA together using FDSOI technology by taking an advantage of a large inductance of a source degenerated LNA input matching network and adding a shunt switch to ground at LNA input to make the LNA appear as a high impedance inductive load for the PA. In addition to FE, Separate standalone PA and LNA test structures were included on the same die to evaluate the effective performance degradation of the front-end implementation at $24-28 \mathrm{GHz}$.

\section{SCHEMATIC}

\section{A. 3-Stack Power Amplifier}

The schematic of the front-end is presented on Fig. 1. The PA is a 3-stack amplifier that allows higher supply voltages up to $2.8 \mathrm{~V}$ from the nominal $0.8 \mathrm{~V}$. The PA is matched with transformers. In the input, the secondary winding of XF1 loaded with $\mathrm{C} 1$ compensates the PA input capacitance and effectively changes the transformer ratio, so that conjugate matching is achieved. The output is matched in similar manner to $\Gamma_{\text {opt }}$. The primary side of XF2 is loaded with $\mathrm{C} 4$ so that the load seen by the PA is down-converted from secondary side impedance. The PA core is inter-stage matched with shunt capacitors $\mathrm{C} 2$ and $\mathrm{C} 3$ at the gates of M1 and M2. 2.8V Supply voltage was isolated from the RF using a $1 \mathrm{nH}$ choke. Transistor core is split into four cells each consisting of five $15 \mu \mathrm{m}$ parallel transistors totaling to width of $300 \mu \mathrm{m}$. Each gate bias of the stack $\left(\mathrm{V}_{\mathrm{g} 1}, \mathrm{~V}_{\mathrm{g} 2}, \mathrm{~V}_{\mathrm{g} 3}\right)$ is controlled via series resistor from off-chip PCB board. Additionally back-gate biases $\left(\mathrm{V}_{\mathrm{bg} 1}, \mathrm{~V}_{\mathrm{bg} 2}, \mathrm{~V}_{\mathrm{bg} 3}\right)$ were controlled in a similar manner.

\section{B. Low Noise Amplifier}

The LNA is a source degenerated cascode, which was designed with minimum noise figure in mind. Circuit design procedure was as follows. First, the input matching network 


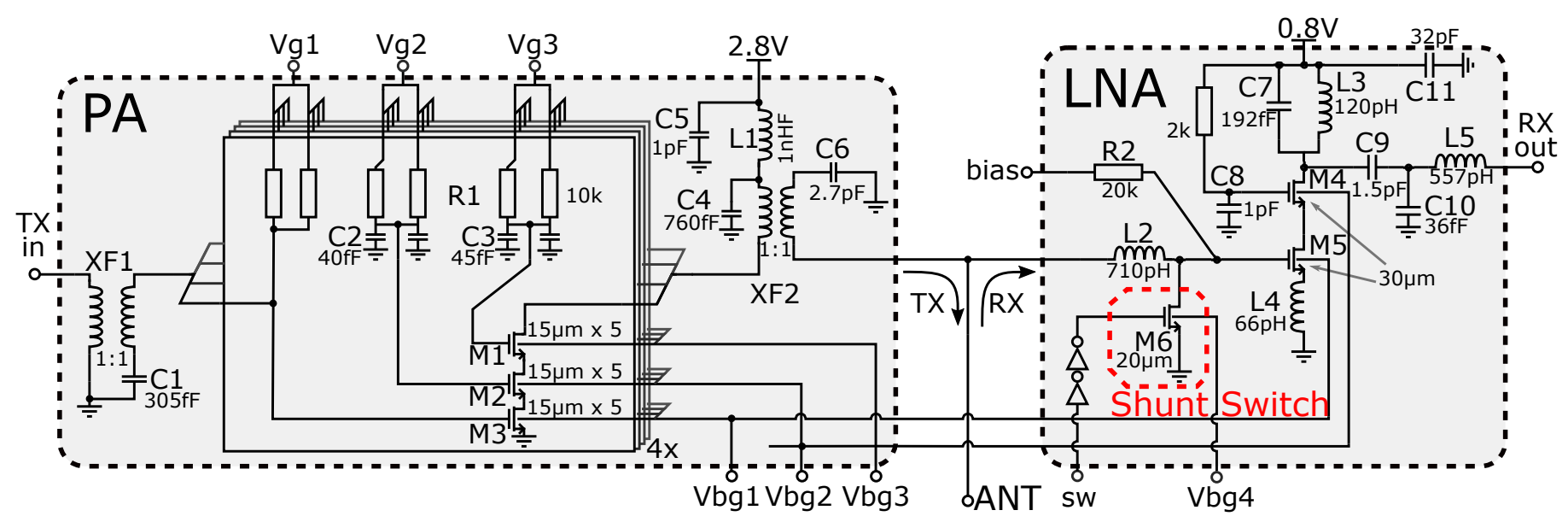

Fig. 1. Front-end schematic.

was designed with some starting values for transistor width and source inductor. Matching is mainly inductive, but a small shunt capacitance was required for optimum noise match. This was implemented together with parasitic capacitances of input stage transistor and with shunt switch transistor. Then the load resonator was designed to have LNA gain peak at $28 \mathrm{GHz}$, and finally the output was matched with an LC-network to $50 \Omega$ including a DC-block capacitor. The transistors are biased close to minimum noise current density of $0.15 \mathrm{~mA} / \mu \mathrm{m}$ or $4.5 \mathrm{~mA}$ with $30 \mu \mathrm{m}$ devices. Stability is ensured with small load to source inductance ratio at a cost of gain.

As mentioned above, all amplifier transistors have backgate connection to study the possible tuning enhancements. Back-gate is simply an additional connection to transistor that allows tuning of the threshold voltage of the transistor with roughly $80 \mathrm{mV} / \mathrm{V}$ sensitivity on this technology [8]. In addition, all capacitors are vertical natural capacitors implemented with lower level metals.

Switch (M6) in the design is controlled to conduct (gate voltage at VDD) in TX mode to protect the LNA gate from large PA output signal swings. The technique is similar to 28nm bulk CMOS implementation in [9] in which differential PA is used. In RX mode the switch is nonconducting and is a part of the matching capacitance of the LNA. Adjusting the VDDs and control signals is done off-chip. In RX mode, the PA VDD is off to minimize any noise that PA generates. Simply switching the biases to $0 \mathrm{~V}$ would break voltage balance of the PA stack resulting in breakdown of gate oxides.

\section{Manufactured Circuits}

Chip micrographs are presented in Fig. 2. Changes between the reference amplifiers and front-end are minimal. In the layout, the LNA input matching inductor L2 had to be modified to connect to a common antenna port. The inductance was increased from $660 \mathrm{pH}$ to $710 \mathrm{pH}$ for optimum performance. Additionally, a DC-block capacitor (C6 in schematic) was added to PA matching network to prevent leakage from LNA bias to ground.

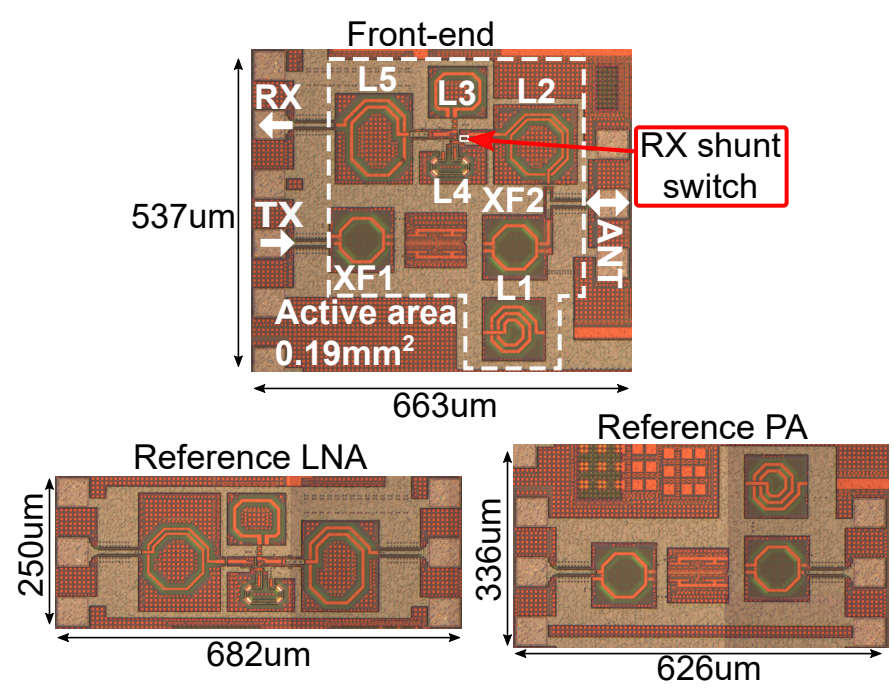

Fig. 2. Front-end, reference LNA and reference PA microphotographs.

\section{MEASUREMENTS}

S-parameters, linearity and noise figure were measured with Keysight PNA-X microwave network analyzer N5247A and a UXA signal analyzer N9040B with noise figure option, noise source 346CK01 and U7228F pre-amplifier. Test chip was probed with Cascade Microtech GSG and GSGSG probes.

\section{A. $R X$}

S-parameters of RX front-end and reference LNA are presented in Fig. 3a. Bias was tuned for maximum gain using $5.8 \mathrm{~mA}$ of quiescent current and then back-gate voltages $\left(\mathrm{V}_{\mathrm{bg} 1}=-0.4 \mathrm{~V} \& \mathrm{~V}_{\mathrm{bg} 2}=0.9 \mathrm{~V}\right)$ were optimized while maintaining the same bias current in order to boost the gain even further.

Off-state PA shifts the center frequency of the LNA from $27.8 \mathrm{GHz}$ to $26.4 \mathrm{GHz}$ that could be tuned back with slight modifications. Peak gain drop of $2 \mathrm{~dB}$ is mainly caused by signal leaking to PA. Noise figure degraded from $3 \mathrm{~dB}$ to $5 \mathrm{~dB}$ at $24 \mathrm{GHz}$. Fig. 3b shows that optimum back-gate bias setup not only increased the gain, but also improved the compression point. This did not have an impact on the noise 

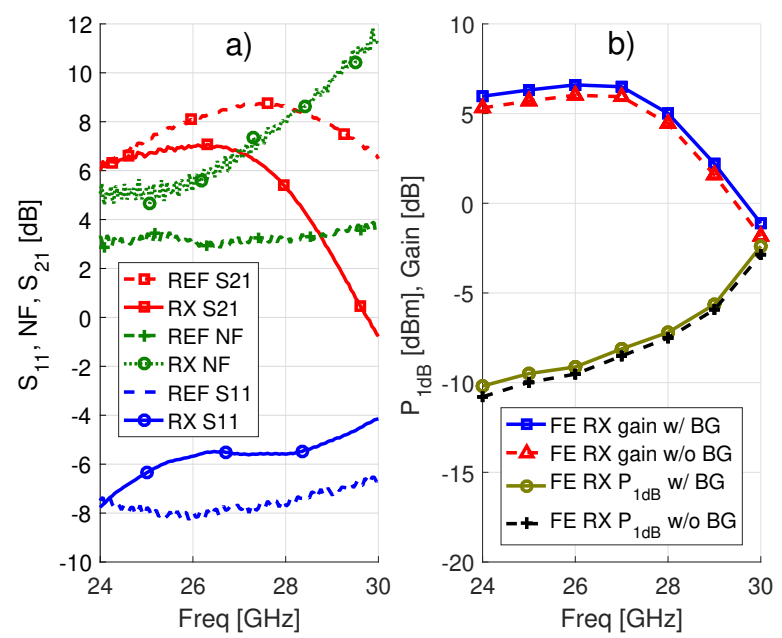

Fig. 3. Measured reference LNA and front-end RX S-parameters and noise figure (a), and gain and compression point with gain optimized backgate biases (b).

figure. Back-bias voltages improve the voltage balance over cascode transistors from $38 \% / 62 \%$ to $51 \% / 49 \%$ in simulations giving more headroom for larger signal swings. Comparing the reference LNA and front-end RX performance, the gain and noise figure degraded $2-5 \mathrm{~dB}$ over $24-28 \mathrm{GHz}$ frequency range. Above $26 \mathrm{GHz}$, part of the receive signal sinks to PA output matching network which resonates around $30 \mathrm{GHz}$ resulting in increase in noise figure.

\section{B. $T X$}

Front-end integration impact on TX performance is limited to additional switch loss which is shown in 1-tone measurements in Fig. 4. Difference in output power is below $2 \mathrm{~dB}$ from $24 \mathrm{GHz}$ to $28 \mathrm{GHz}$ indicating decent de-Q and bandwidth extension due to the switch. Wide TX bandwidth indicates a possibility to tune the TX matching network to a higher frequency improving noise figure degradation without additional TX penalty. Due to the switch loss, peak PAE decreased from $23 \%$ to $10 \%$ showing a good efficiency among
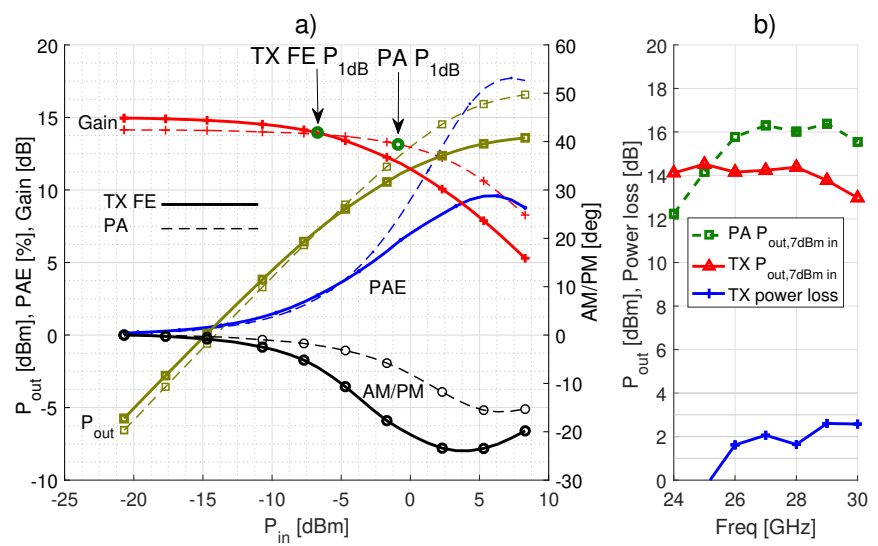

Fig. 4. Measured 1-tone compression, AM-PM and PAE of TX FE and reference $\mathrm{PA}$ at $28 \mathrm{GHz}(\mathrm{a})$, and $\mathrm{P}_{\text {sat }}$ and $\mathrm{TX}$ loss over frequency (b).
Ka-band CMOS front-ends. The non-linear load, M6, has impact on compression impacting AM/PM distortion at lower output power levels that can be compensated with proper linearization.

\section{Modulated Measurements}

TX performance was tested with $100 \mathrm{MHz}$ wide cyclic prefix - orthogonal frequency division multiplexing (CP-OFDM) 64-QAM modulated signal following the 3GPP/NR standard for FR2. The used waveform has peak-to-average-power-ratio (PAPR) of $10.9 \mathrm{~dB}$ due to the OFDM. Signal was generated with Keysight M8190A Arbitrary waveform generator and upconverted to $28 \mathrm{GHz}$ by Keysight E8267E signal generator. The output was measured
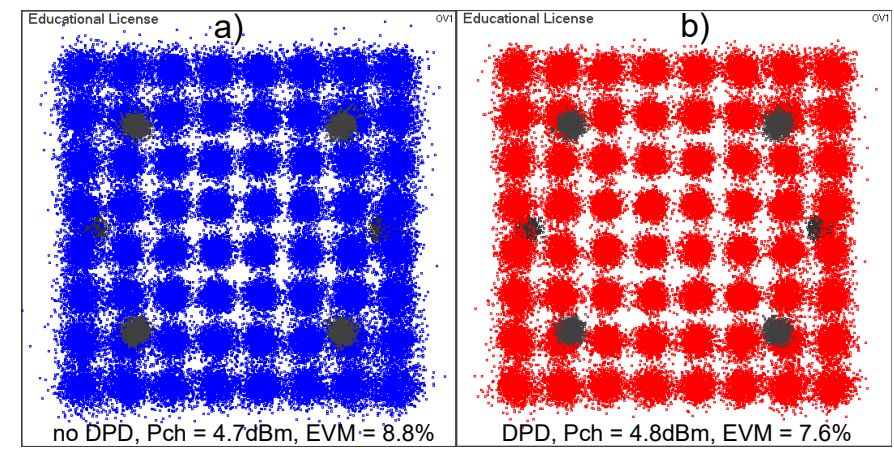

Fig. 5. Measured constellations without (a) and with DPD (b).

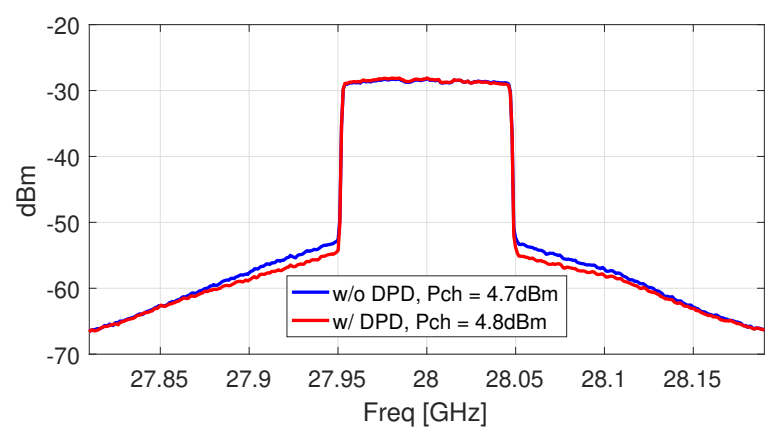

Fig. 6. Measured output spectrums with (red) and without DPD (blue) with $4.8 \mathrm{dBm}$ output power.

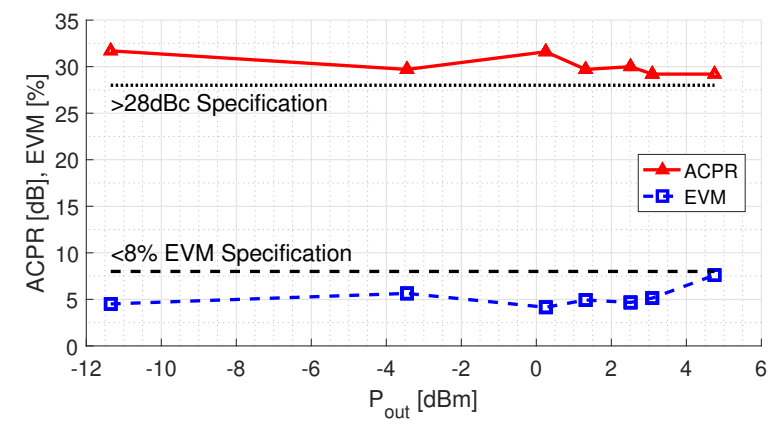

Fig. 7. Measured output channel power, ACPR and EVM as function of output power with DPD and 5G specifications from [12] for $100 \mathrm{MHz}$ 64-QAM signal. 
Table 1. Comparison of mmWave TDD front-ends.

\begin{tabular}{|c|c|c|c|c|c|c|c|c|}
\hline Reference & $\begin{array}{c}\text { This } \\
\text { work }\end{array}$ & [9] & {$[4]$} & [5] & [6] & {$[10]$} & [11] & [7] \\
\hline Switch type & \multicolumn{2}{|c|}{ RX shunt gate } & SPDT & $1 / 4 \lambda \mathrm{TL}$ & \multicolumn{4}{|c|}{ Common match } \\
\hline Freq [GHz] & $24-28$ & $25-30.5$ & $30-40$ & $27-29$ & $57-65$ & $27-40$ & $60-67$ & $25-30$ \\
\hline RX gain $[\mathrm{dB}]$ & 7 & $34 *$ & 17 & $30 *$ & 21.5 & 16.1 & 17 & 11 \\
\hline RX P1dB [dBm] & -9.1 & - & -17 & $-22.5^{*}$ & - & -15.7 & -25 & -7.1 \\
\hline RX NF [dB] & 5 & $3.8 *$ & $8.4(\mathrm{sim})$ & 6 & 6.7 & 6.2 & 6.8 & 3.5 \\
\hline Pdiss RX [mW] & 4.6 & $42 *$ & 48 & $103.1^{*}$ & 39.6 & 17.6 & $<28.8$ & 28 \\
\hline TX gain [dB] & 15 & $44 *$ & 14 & 15 & 24.5 & 28.5 & 24 & 12 \\
\hline TX P1dB [dBm] & 7.4 & $12 *$ & 20.5 & 13.5 & 5 & 14.1 & 10 & 22 \\
\hline Pdiss TX [mW] & 183 & $90 *$ & 352 & $143.8^{*}$ & 71 & 96.2 & $<63.5$ & 230 \\
\hline Psat $[\mathrm{dBm}]$ & 13.6 & 14 & 22.5 & 16 & 8.4 & 15.8 & 10 & 23.6 \\
\hline PAE [\%] & 9.6 & 20 & 7 & 21 & 8.7 & 20 & - & 28 \\
\hline Active area $\left[\mathrm{mm}^{2}\right]$ & 0.19 & $1.16^{*}$ & $7 *$ & 4 & 0.22 & 0.35 & 0.35 & 0.275 \\
\hline Process & 22nm SOI CMOS & 28nm CMOS & $130 \mathrm{~nm}$ & SiGe & $65 \mathrm{~nm}$ & $\overline{\mathrm{CMOS}}$ & 28nm CMOS & 45nm SOI CMOS \\
\hline
\end{tabular}

*complete TX/RX chain

with Keysight N9040B UXA. To meet the 3GPP/NR FR2 specifications of $8 \% \mathrm{EVM}$ and $28 \mathrm{dBc}$ ACPR, digital predistortion (DPD) was applied to the signal [12]. Output constellation diagrams and spectrums with and without DPD are shown in Fig. 5 and Fig. 6 respectively. Maximum channel power is $4.8 \mathrm{dBm}$ indicating a realistic $9 \mathrm{~dB}$ backoff for the used waveform which mean that the waveform peaks are already almost $2 \mathrm{~dB}$ in saturation. ACPR and EVM characteristics over measured output power range are shown in Fig. 7. EVM starts to deteriorate when the back-off is over $11 \mathrm{~dB}$, close to the waveform PAPR.

\section{CONCLUSION}

The front-end is compared to reported state-of-the-art front-ends with various switch topologies in Table 1. This is the first front-end implemented on 22nm FDSOI technology achieving lowest area and RX power dissipation, while providing competitive output power, efficiency and receive noise figure. Measurements with real 5G NR test signals show capability to support even 64-QAM OFDM signals with 9 $\mathrm{dB}$ backoff. Simple design minimizes workload in front-end co-design. At $24 \mathrm{GHz}, \mathrm{RX}$ and TX performance is degraded only by $2 \mathrm{~dB}$ which is only possible to achieve using a switch topology with decent isolation. Output power and receive noise figure can be further improved by more careful PA load impedance tuning and adding more transistors to PA stack.

\section{ACKNOWLEDGMENT}

This research has been financially supported by Academy of Finland 6Genesis Flagship (grant 318927) and Nokia Corporation Ltd. GLOBAL FOUNDRIES is acknowledged for silicon fabrication and technical support.

\section{REFERENCES}

[1] S.N. Ong, S. Lehmann, W.H. Chow, C. Zhang, et al., "A 22nm FDSOI Technology Optimized for RF/mmWave Applications," in Proc. IEEE RFIC Symposium, Philadelphia, PA, USA, Jun. 2018, pp. 72-75.
[2] O. El-Aassar and G. M. Rebeiz, "A Compact DC-to-108GHz Stacked-SOI Distributed PA/Driver Using Multi-Drive InterStack Coupling, Achieving 1.525THz GBW, 20.8dBm Peak P1dB, and Over $100 \mathrm{~Gb} / \mathrm{s}$ in 64-QAM and PAM-4 Modulation," in ISSCC, San Francisco, CA, USA, Feb. 2019, pp. 86-87.

[3] P. M. Asbeck, N. Rostomyan, M. Özen, B. Rabet and J. A. Jayamon, "Power Amplifiers for mm-Wave 5G Applications: Technology Comparisons and CMOS-SOI Demonstration Circuits," IEEE Trans. Microw. Theory Techn., vol. 67, no. 7, pp. 3099-3109, Feb. 2019.

[4] C.Liu, Q. Li, Y. Li, X. Deng, H. Tang, R. Wang, H. Liu and Y. Xiong, "A Ka-Band Single-Chip SiGe BiCMOS Phased-Array Transmit/Receive Front-End," IEEE Trans. Microw. Theory Techn., vol. 64, no. 11, pp. 3667-3677, Sep. 2016.

[5] B. Sadhu, Y. Tousi, J. Hallin and S. Sahl, "A 28-GHz 32-Element TRX Phased-Array IC With Concurrent Dual-Polarized Operation and Orthogonal Phase and Gain Control for 5G Communications," IEEE J. Solid-State Circuits, vol. 52, no. 12, pp. 3373-3391, Dec. 2017.

[6] F. Meng, K. Ma, K. S. Yeo, C. C. Boon, X. Yi, J. Sun, G. Feng and S. Xu, "A Compact 57-67 GHz Bidirectional LNAPA in 65-nm CMOS Technology," IEEE Microw. Wireless Compon. Lett., vol. 26, no. 8, pp. 628-630, Jul. 2016.

[7] N. Rostomyan, M. Özen, P. M. Asbeck, "Synthesis Technique for Low-Loss mm-Wave T/R Combiners for TDD Front-Ends," IEEE Trans. Microw. Theory Techn., vol. 67, no. 3, pp. 1030-1038, Mar. 2019.

[8] A. Majumdar, X. Wang, A. Kumar, J. R. Holt, et al., "Gate Length and Performance Scaling of Undoped-Body Extremely Thin SOI MOSFETs," IEEE Electron Device Lett., vol. 30, no. 4, pp. 413-415, Feb. 2009.

[9] J. D. Dunworth, A. Homayoun, B-H. Ku, Y-C. Ou, et al., "A $28 \mathrm{GHz}$ Bulk-CMOS dual-polarization phased-array transceiver with 24 channels for 5G user and basestation equipment," pp. 70-71, Feb. 2018.

[10] S. Mondal, R. Singh, J. Paramesh, "A Reconfigurable Bidirectional 28/37/39GHz Front-End Supporting MIMO-TDD, Carrier Aggregation TDD and FDD/Full-Duplex with Self-Interference Cancellation in Digital and Fully Connected Hybrid Beamformers," in ISSCC, San Francisco, CA, USA, Feb. 2019, pp. 348-349.

[11] K. Khalaf, K. Vaesen, S. Brebels, G. Mangraviti, M. Libois, C. Soens and P. Wambacq, "A $60 \mathrm{GHz} 8$-way phased array front-end with TR switching and calibration-free beamsteering in 28nm CMOS," in ESSCIRC, Leuven, Belgium, Sep. 2017.

[12] 3GPP, "Base Station (BS) radio transmission and reception," 3rd Generation Partnership Project (3GPP), Technical Specification (TS) 38.104, 10 2019, version 16.1.0. [Online]. Available: https://www.3gpp.org/ftp/Specs/archive/38_series/38.104/38104-g10.zip 